\title{
Camptodactyly-taurinuria syndrome
}

INSERM

\section{Source}

INSERM. (1999). Orphanet: an online rare disease and orphan drug data base.

Camptodactyly-taurinuria syndrome. ORPHA:1325

Camptodactyly-taurinuria syndrome is a congenital malformation syndrome characterized by the association of a permanent camptodactyly of the fingers (see this term) with the over excretion of taurine in the urine. Camptodactyly mainly affects the little finger, although any finger may be involved. The disease has been described in 17 affected patients from 4 unrelated families. An autosomal dominant inheritance has been suggested. There have been no further descriptions in the literature since 1966. 